\title{
Overexpression of long non-coding RNA cancer susceptibility 2 inhibits cell invasion and angiogenesis in gastric cancer
}

\author{
JIANFENG ZHOU, HAIYAN HUANG, SHIJIE TONG and RONG HUO \\ Department of Clinical Medicine, Xiaogang Hospital, Ningbo, Zhejiang 315800, P.R. China
}

Received October 25, 2016; Accepted June 13, 2017

DOI: $10.3892 / \mathrm{mmr} .2017 .7233$

\begin{abstract}
Increasing evidence has indicated that long non-coding RNAs (lncRNAs) were aberrantly expressed and acted as key regulators in various types of disease, including cancer. IncRNA cancer susceptibility 2 (CASC2) has been found to be downregulated and acts as a tumor suppressor in various type of cancer, including gastric cancer (GC). However, the precise function of IncRNA CASC2 in GC remains unclear. In the present study, the expression level of lncRNA CASC2 in GC was investigated and the molecular mechanisms by which CASC2 acted as a tumor suppressor in this disease were elucidated. It was found that the expression level of 1ncRNA CASC2 was decreased, which correlated with TNM stages, vessel invasion, metastasis, and overall survival of patients with GC. Furthermore, overexpression of CASC2 inhibited the invasion and angiogenesis of GC cells. Thus, the present study indicated the important roles and underlying molecular mechanisms of 1ncRNA CASC2 on GC, and indicated that IncRNA CASC 2 may present as a potential therapeutic target for the treatment of GC.
\end{abstract}

\section{Introduction}

Gastric cancer (GC) is the fourth most commonly diagnosed type of cancer and the second leading cause of global cancer-associated mortality worldwide $(1,2)$. Although advances in surgical techniques, radiotherapy and chemotherapy have been made in the treatment of GC over the past two decades, the prognosis for GC patients remains poor due to many patients being diagnosed at an advanced stage with extensive invasion and lymphatic metastasis, and the majority develop regional or distant recurrences $(3,4)$. Previous studies have identified many genes closely associated with GC progress, although the underlying complex molecular mechanisms remain obscure. Therefore, an improved understanding of

Correspondence to: Dr Haiyan Huang, Department of Clinical Medicine, Xiaogang Hospital, 368 South East Road, Ningbo, Zhejiang 315800, P.R. China

E-mail: zhuxufifa2007@163.com

Key words: long non-coding RNA cancer susceptibility 2, gastric cancer, proliferation, migration the molecular mechanisms underlying GC may improve the diagnosis and therapy of patients with GC.

Long non-coding RNAs (lncRNAs) are a class of single-stranded RNA molecules $>200$ nucleotides without protein-coding capacity $(5,6)$. Increasing evidence demonstrated that lncRNAs are critical in multiple biological processes, including cell growth, cell differentiation, cell apoptosis and cell invasion (7-10). For example, it has been found that lncRNA-HOX transcript antisense RNA was upregulated in diffuse large B cell lymphoma, and correlated with an invasive phenotype and poor prognosis (7). Furthermore, a reduction of 1ncRNA GAS5 was identified as an independent prediction factor for overall survival (OS; calculated from the date of surgery to the date of death due to any cause or last follow-up), and overexpression of GAS5 suppressed the cell proliferation and invasion ability (8).

The IncRNA, small nucleolar RNA cancer susceptibility 2 (CASC2), located at chromosome 10q26, was originally identified as a downregulated gene and acted as a tumor suppressor gene in endometrial cancer (11). CASC2 was downregulated in glioma tissues, and overexpression of CASC2 inhibited cell proliferation, migration and invasion via negative regulation of miR-21 (12). In a recent study, it was indicated that lncRNA CASC2 expression levels were significantly decreased in GC (13); however, the clinical significance and biological functions of CASC2 in GC remain unclear.

The present study first determined the expression and clinicopathological features of CASC2 in GC tissue, and then investigated its biological functions and associated mechanisms by overexpression of CASC2.

\section{Materials and methods}

Patient samples. A total of 69 fresh GC tissue specimens, together with matched adjacent non-tumorous tissue specimens, were collected from patients who underwent surgery at the Xiaogang Hospital (Ningbo, China) between May 2011 and June 2012. Written consent to use the samples for research was obtained from all patients prior to surgery. None of the patients received preoperative therapy prior to surgical resection. The histologic diagnosis of tumors was performed by at least two experienced pathologists based on the World Health Organization criteria (14). All samples were immediately frozen following resection in liquid nitrogen and stored at $-80^{\circ} \mathrm{C}$ until RNA extraction. The study was approved by the 
A

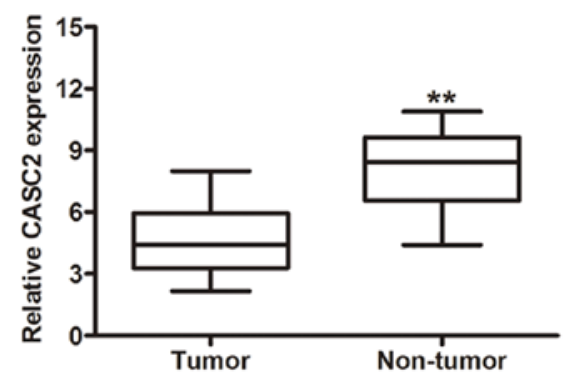

B

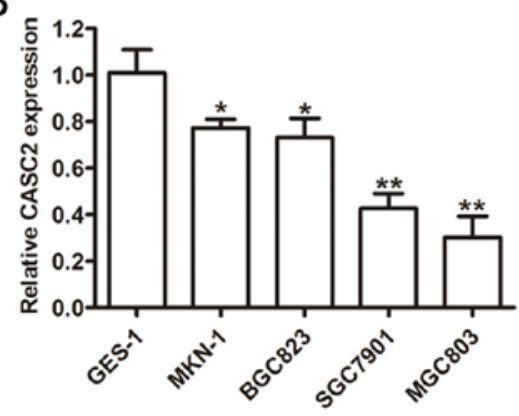

Figure 1. Long none-coding RNA CASC2 was downregulated in GC tissue samples and GC cell lines. (A) Relative expression levels of CASC2 in human GC tissue samples compared with corresponding non-tumor tissue samples $(n=69)$. (B) Reverse transcription-quantitative polymerase chain reaction analysis of CASC2 expression levels in GC cell lines compared with the normal bronchial epithelial cell line (GES-1). GAPDH served as the internal control. * $<0.05$, ${ }^{* * *} \mathrm{P}<0.01$. CASC2, cancer susceptibility 2; GC, gastric cancer.

Institutional Review Board and Human Ethics Committee of Xiaogang Hospital.

Cell culture. Human umbilical vein endothelial cells (HUVECs), 4 human GC cell lines (MKN-1, SGC7901, BGC823 and MGC803) and the nonmalignant epithelial gastric cell line, GES-1 were obtained from the Shanghai Cell Bank of the Chinese Academy of Sciences (Shanghai, China). All cells were cultured in Dulbecco's modified Eagle's medium (HyClone; GE Healthcare Life Sciences, Logan, UT, USA) containing 10\% fetal bovine serum (Invitrogen; Thermo Fisher Scientific, Inc., Waltham, MA, USA), $100 \mathrm{U} / \mathrm{ml}$ penicillin, $100 \mu \mathrm{g} / \mathrm{ml}$ streptomycin at $37^{\circ} \mathrm{C}$ in a humidified atmosphere of $5 \% \mathrm{CO}_{2}$.

Construct generation and transient transfection. IncRNA-CASC 2 cDNA was constructed by cloning the cDNA sequence of CASC2 into the pcDNA3.1 expression vector (Invitrogen; Thermo Fisher Scientific, Inc.) by Shanghai Furui Science \& Technology Co., Ltd. (Shanghai, China). For the transfection of the pcDNA-CASC2, GC cells were transfected with control vector or pcDNA-CASC2 constructs at a final concentration of $1 \mu \mathrm{g} / \mu \mathrm{l}$ using Lipofectamine ${ }^{\circledR} 2000$ Reagent (Invitrogen; Thermo Fisher Scientific, Inc.) according to the manufacturer's protocol. Following transfection for $48 \mathrm{~h}$, cells were harvested for RT-qPCR analysis and the subsequent experiments.

Cell proliferation analysis. The capability of cellular proliferation was assessed via Cell Counting Kit-8 assay (Dojingdo Molecular Technologies, Inc., Kumamoto, Japan). Approximately $3 \times 10^{3}$ cells transfected with control vector or CASC2 were seeded into 96-well culture plates. CCK-8 solution $(10 \mu \mathrm{l})$ was added and incubated at $37^{\circ} \mathrm{C}$ for $2 \mathrm{~h}$ and maintained for 24, 48, 72 and $96 \mathrm{~h}$. Finally, the absorbance at a wavelength of $450 \mathrm{~nm}$ was measured using a spectrophotometer reader (Elx800; BioTek Instruments, Inc., Winooski, VT, USA). All experiments were performed as 5 replicates and were repeated 3 times independently.

RNA extraction, reverse transcription-quantitative polymerase chain reaction $(R T-q P C R)$. Total RNA from the frozen samples and cell lines was extracted using Invitrogen TRIzol reagent; Thermo Fisher Scientific, Inc.) according to the manufacturer's protocol. cDNA from all samples was synthesized from 500 ng total RNA using a PrimeScript ${ }^{\circledR} 1$ st strand cDNA Synthesis kit (Takara Biotechnology Co., Ltd., Dalian, China) on an ABI 9700 DNA thermal cycler (Applied Biosystems; Thermo Fisher Scientific, Inc.) following the manufacturer's protocols. qRT-PCR analyses were performed using an SYBR Premix EX Taq ${ }^{\mathrm{TM}}$ II kit (Biotechnology Co., Ltd.) on the ABI Prism 7900. The thermal cycling conditions involved initial denaturation for $10 \mathrm{sec}$ at $94^{\circ} \mathrm{C}$, followed by 40 cycles of $5 \mathrm{sec}$ at $94^{\circ} \mathrm{C}$ and $30 \mathrm{sec}$ at $60^{\circ} \mathrm{C}$. The PCR primers for CASC2 and glyceraldehyde-3-phosphate dehydrogenase (GAPDH) were as follows: Forward, 5'-GCTGATCAGAGC ACATTGGA-3' and reverse 5'-ATAAAGGTGGCCACAACT GC-3' for CASC2; forward, 5'-ACCCACTCCTCCACCTTT GAC-3' and reverse, 5'-TGTTGCTGTAGCCAAATTCGTT-3' for GAPDH. The expression level of CASC2 was calculated using the $2^{-\triangle \Delta C q}$ method and normalized to the respective GAPDH expression level (15). The specificity of each PCR reaction was confirmed by melting curve analyses.

Cell apoptosis analysis. For the apoptosis assays, cells were harvested with trypsin enzyme and washed with phosphate-buffered saline $48 \mathrm{~h}$ after transfection. Annexin-V-fluorescein isothiocyanate and propidium iodide (BD Biosciences, Franklin Lakes, NJ, USA) were added to stain the cells according to the manufacturer's protocols. Finally, Apoptotic cells were measured with a Beckman Coulter FC500 (Beckman Coulter, Inc., Brea, CA, USA) and analyzed using FlowJo version 10.07 (FlowJo LLC, Ashland, OR, USA).

Endothelial tube formation in vitro. GC cells were transfected with control vector or pcDNA-CASC2. After transfection (48 h), the supernatants were collected and centrifuged at $14,000 \mathrm{x} \mathrm{g}$ for $30 \mathrm{~min}$ at $4^{\circ} \mathrm{C}$ to discard cell debris. The supernatant was harvested for further investigations. A tube formation assay was performed as previously described (16). HUVECs were cultured in conditioned medium from the supernatant of the GC cells at a density of 20,000 cells per well in a 96-well plate precoated with $150 \mu \mathrm{l}$ Matrigel (BD Biosciences, Franklin Lakes, NJ, USA). The HUVECs were cultured at $37^{\circ} \mathrm{C}$ in a $5 \%$ $\mathrm{CO}_{2}$ atmosphere for $6 \mathrm{~h}$. The formation of HUVEC tubular structures was calculated and imaged under a light microscope (Olympus Corp., Tokyo, Japan) at a magnification of x100. 
Table I. Correlation between lncRNA CASC 2 expression and clinicopathological factors in gastric cancer patients.

\begin{tabular}{lccc}
\hline & \multicolumn{2}{c}{$\begin{array}{l}\text { IncRNA CASC2 } \\
\text { expression level }\end{array}$} & \\
\cline { 2 - 3 } Variable & Low (n=35) & High (n=34) & P-value \\
\hline Gender & & & 0.8075 \\
Female & 13 & 14 & \\
Male & 22 & 20 & \\
Age, years & & & 0.8106 \\
$\leq 60$ & 17 & 15 & \\
$>60$ & 18 & 19 & \\
Tumor site & & & 0.2104 \\
Cardia & 6 & 4 & \\
Body & 12 & 17 & \\
Antrum & 17 & 13 & 0.0160 \\
Vessel invasion & & & \\
Positive & 24 & 13 & \\
Negative & 11 & 21 & \\
Differentiation & & & \\
Well/moderately & 21 & 26 & \\
Poorly & 14 & 8 & 0.1975 \\
TNM stage & & 31 & \\
I-II & 20 & 30 & \\
III-IV & 15 & 4 & \\
Metastasis & & & \\
Positive & 11 & 3 & \\
Negative & 24 & 310063 \\
\hline
\end{tabular}

lncRNA, long non-coding RNA; CASC2, cancer susceptibility 2.

Cell invasion assay. To assess the cell motility ability in vitro, transwell chambers with $8-\mu \mathrm{m}$ pore polycarbonate membranes (Corning Incorporated, Corning, NY, USA) were used. Following transfection (24 h), cells were collected and re-suspended in $100 \mu \mathrm{l}$ serum-free medium, and seeded on the Matrigel-coated upper chamber. The lower chamber contained complete medium as a chemoattractant. Following $48 \mathrm{~h}$ incubation at $37^{\circ} \mathrm{C}$, the cells on the lower side of the membrane were fixed and stained with $0.5 \%$ crystal violet solution (Beyotime Institute of Biotechnology, Haimen, China). Five fields of vision were randomly selected and cell numbers were counted under a light microscope (Olympus Corp.).

Statistical analysis. All data are expressed as the mean \pm standard deviation of at least three independent experiments. The difference between two groups was estimated using Student's t-test. The $\chi^{2}$ test was used to assess the association between the expression of CASC 2 and clinicopathological features. The OS rates were analyzed using the Kaplan-Meier method, with the log-rank test applied for comparison. All statistical analyses were performed using SPSS version 11.5 (SPSS, Inc., Chicago, IL, USA). P<0.05 was considered to indicate a statistically significant difference.

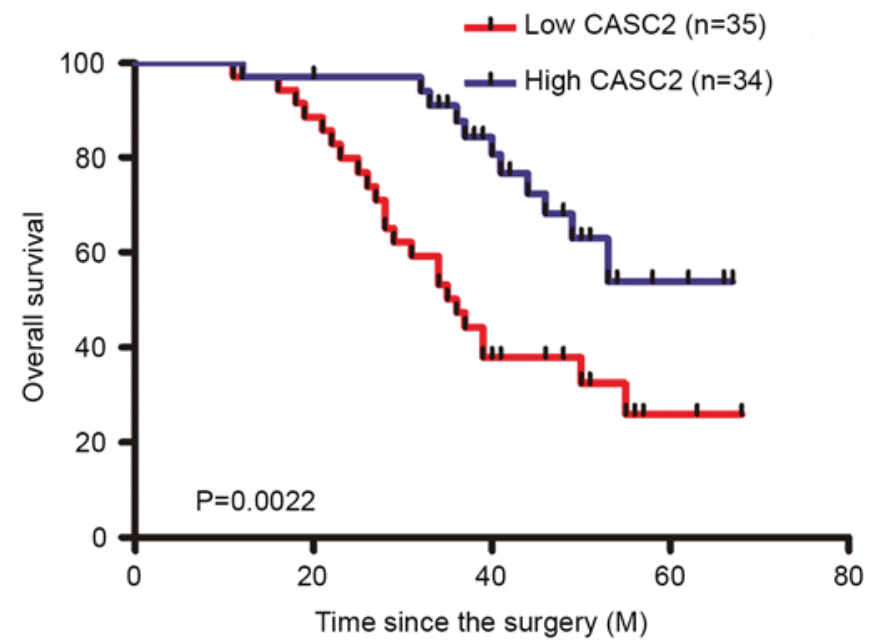

Figure 2. Kaplan-Meier curves of overall survival of patients with gastric cancer, according to CASC2 expression level. CASC2, cancer susceptibility 2.

\section{Results}

IncRNA CASC2 expression levels decreased in GC samples and cell lines. Previous studies indicated that the expression level of CASC2 was decreased in GC tissues and colorectal cancer $(13,17)$. The expression level of IncRNA CASC2 was initially confirmed in GC tissue samples and the results indicated that the relative expression of CASC 2 in GC tissue was significantly decreased compared with the non-tumor samples (Fig. 1A). The CASC2 expression level was markedly decreased in the GC cell lines compared with the normal gastric epithelial cell line, GES-1 (Fig. 1B). As SGC7901 and MGC803 exhibited relatively low endogenous CASC2 expression levels among all of the tested cell lines, these two cell lines were selected for the subsequent experiments.

Low CASC2 expression levels correlated with poorer survival. In order to establish whether the expression level of CASC2 in GC tissues exerted an effect on patient clinicopathological features, the correlation between CASC 2 expression level and clinicopathological features were further assessed. The 69 primary GC patients were divided into two groups based on the mean value of CASC2 in tumor tissue samples: A high-CASC2 group ( $n=34$; CASC2 expression ratio >median) and a low-CASC2 group $(n=35$; CASC2 expression ratio $\leq$ median). The associations between CASC2 expression level and the clinicopathological features are summarized in Table I. Notably, low CASC2 expression levels in GC samples were significantly correlated with vessel invasion $(\mathrm{P}=0.016)$, advanced TNM stage $(\mathrm{P}=0.0063)$ and metastasis $(\mathrm{P}=0.034)$. However, no significant difference between CASC2 expression level and sex, age, tumor size, cell differentiation, or tumor site was identified. To determine the association between CASC2 expression level and the prognosis of GC patients, OS curves were plotted according to the CASC2 expression level and analyzed using the Kaplan-Meier method and log-rank test. The high-CASC2 expression group had significantly shorter OS than the low-CASC2 expression group (Fig. 2). These results indicate that CASC2 may present as a useful marker of the prognosis or progression of GC. 


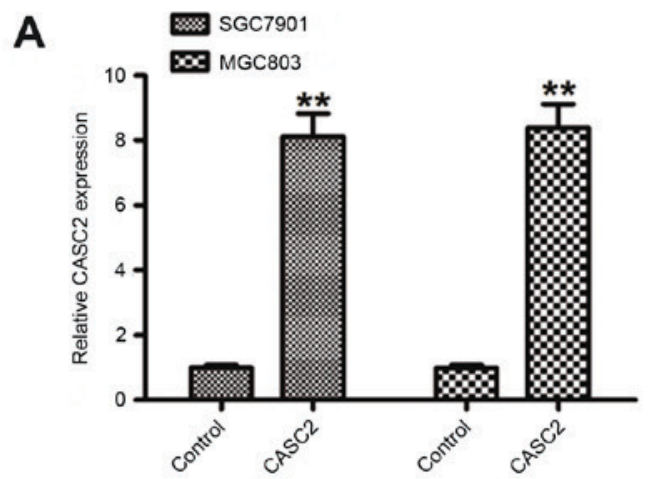

B
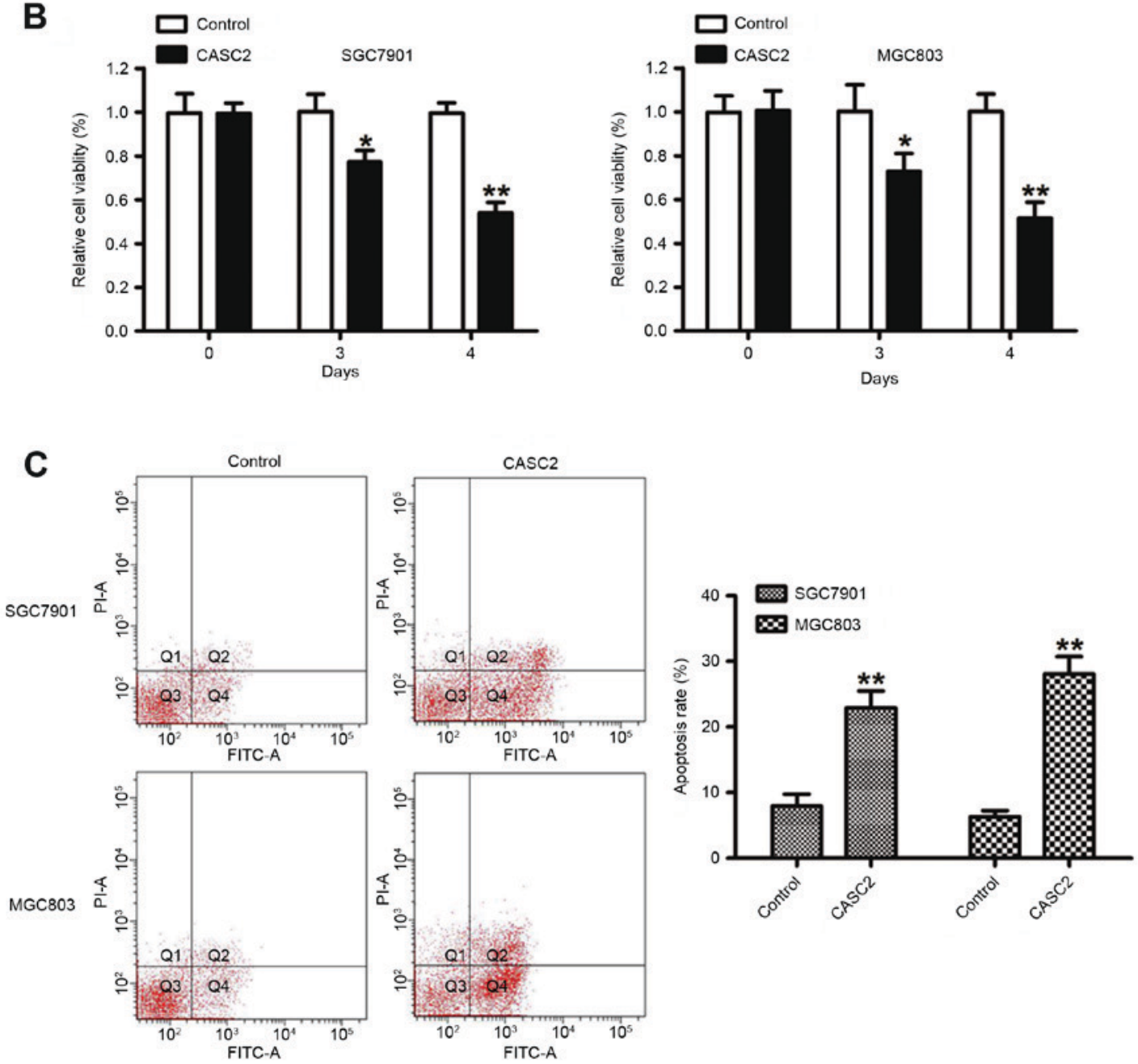

Figure 3. Effect of CASC2 on GC cell proliferation. (A) Reverse transcription quantitative polymerase chain reaction analysis of CASC2 expression levels in SGC7901 and MGC803 cells transfected with control vector or CASC2. (B) Cell Counting Kit 8 assays were performed to determine the proliferation of GC cells transfected with control vector or CASC2. (C) Apoptosis in SGC7901 and MGC803 cells following CASC2 overexpression was detected by flow cytometry. ${ }^{*} \mathrm{P}<0.05,{ }^{* *} \mathrm{P}<0.01$. CASC2, cancer susceptibility 2; GC, gastric cancer; PI, propidium iodide; FITC, fluorescein isothiocyanate.

Overexpression of CASC2 inhibits GC cell proliferation in vitro. To assess the biological role of CASC2 in GC, the effect of CASC2 on GC cell lines was evaluated. CASC2 was overexpressed by transfecting the pcDNA3.1-CASC2 vector into the SGC7901 and MGC803 cell lines, which contained the lowest expression level of CASC2. RT-qPCR analysis was performed $48 \mathrm{~h}$ post-transfection and the data revealed that ectopic overexpression of CASC2 significantly increased the expression level of CASC2 in SGC7901 and MGC803 cell lines (Fig. 3A). To further identify the function of CASC2 on cell growth, cell proliferation and colony formation assays were performed. As shown in Fig. 3B, cell proliferation was significantly inhibited when CASC2 was overexpressed in the SGC7901 and MGC803 cells. Furthermore, compared with the control vector, CASC2-overexpressing SGC7901 and MGC803 cells markedly induced cell apoptosis. These data indicated that CASC2 acted as a tumor suppressor by suppressing proliferation and inducing GC cells. 

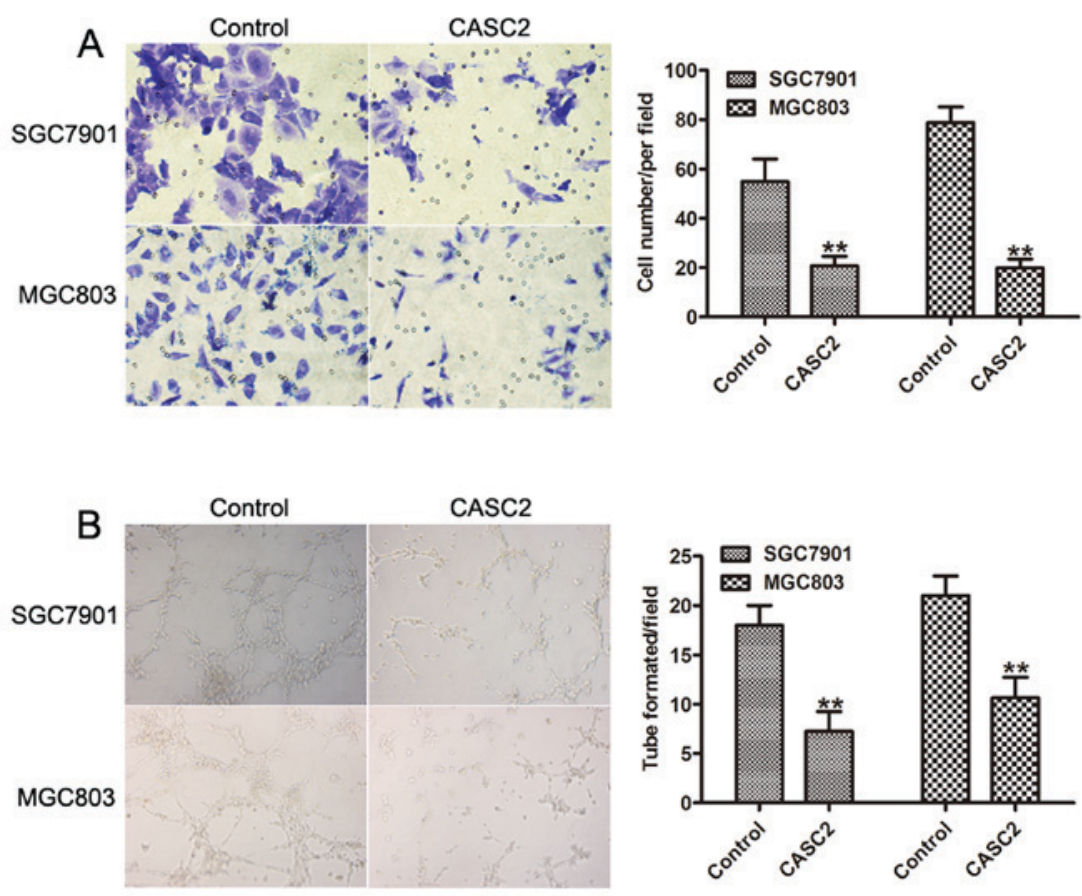

Figure 4. Overexpression of CASC2 suppressed invasion and angiogenesis of GC cells. (A) Transwell assays were performed to investigate the migratory abilities of the control vector or the pcDNA-CASC2-transfected GC cells. Magnification, x200. (B) Representative images of tube-like structures of human umbilical vein endothelial cells cultured with conditioned medium derived from the control vector or CASC2-transfected GC cells were used for the Matrigel tube formation assay. ${ }^{* *} \mathrm{P}<0.01$. CASC2, cancer susceptibility 2 ; GC, gastric cancer.

Overexpression of CASC2 inhibits GC cell invasion and angiogenesis. As a significant difference between the expression level of IncRNA CASC2, and vessel invasion and metastasis was identified, whether CASC2 is involved in GC cell invasion was then investigated. The invasion assay indicated that overexpression of 1ncRNA CASC2 significantly inhibited cell invasion in SGC7901 and MGC803 cells (Fig. 4A). Furthermore, the results demonstrated that formation of capillary-like structures of HUVECs treated with CASC2-conditioned medium were significantly decreased (Fig. 4B). These results revealed that CASC2 acted as a tumor suppressor via inhibiting cell invasion and angiogenesis.

\section{Discussion}

Various studies revealed that $>10,000$ lncRNAs are encoded by the human genome in integrative genomic analyses (18-20). Despite rapid progress in screening, cancer-associated lncRNAs were observed to be involved in the development of cancer $(16,18)$. Recent findings indicate that many lncRNAs act as oncogenes or tumor suppressors, and are important in GC (13,21,22). H19, HULC, and SPRY4-IT1 were identified to increase in GC tissue samples and their knockdown strongly inhibited proliferation, migration and invasion of GC cells in vitro $(23-25)$.

CASC2 was identified to be significantly downregulated in GC tissue samples and overexpression of CACS2 may inhibit GC cell proliferation via inactivation of the extracellular signal-regulated kinase $1 / 2$ and c-Jun N-terminal kinase/mitogen-activated protein kinase signaling pathways (13). However, the mechanisms underlying the aberrant expression of CACS2 in GC remain elusive. Consistent with previous studies, the present study indicated that the expression of CACS2 in GC tissue samples was significantly higher than that in corresponding non-tumor tissue samples. Furthermore, statistical analyses revealed that a low expression level of CACS2 in GC samples was associated with the depth of invasion, TNM stage and metastasis. Low CACS2 expression levels in GC tissues were associated with a short OS and may be an independent prognostic factor. These results indicate that CACS2 may function as a tumor suppressor in GC progression. Furthermore, it was identified that overexpression of CACS2 inhibited tumor cell proliferation by inducing cell apoptosis.

Transwell assay revealed that the overexpression of CACS2 decreased cell motility and angiogenesis. The present study identified a GC-associated lncRNA, CACS2, and elucidated its functional roles in GC development and progression. However, there is a lack of information regarding the underlying mechanism of CACS2; therefore, more experimental studies on CACS2 are required to provide important information to further our understanding of its functional roles in GC.

In conclusion, the present study indicates that CACS2 expression levels were significantly decreased in GC tissue samples when compared with healthy adjacent tissue samples, as well as GC cell lines. In addition, a low CACS2 expression level was associated with poor prognosis and may present as a negative prognostic factor for GC patients. The current data demonstrate that the migration and invasion ability of GC cells were significantly inhibited by the overexpression of CACS2, indicating CACS2 as an important regulator of metastasis. Thus, these data indicated an important role of CACS2 in the molecular etiology of GC and implicate the potential application of CACS2 in GC therapy. 


\section{Acknowledgements}

The present study was supported by grants from Ningbo Science and Technology Project (grant nos. 2015A610316, 201501CX-D02057)

\section{References}

1. Torre LA, Bray F, Siegel RL, Ferlay J, Lortet-Tieulent J and Jemal A: Global cancer statistics, 2012. CA Cancer J Clin 65: 87-108, 2015

2. Herszenyi L and Tulassay Z: Epidemiology of gastrointestinal and liver tumors. Eur Rev Med Pharmacol Sci 14: 249-258, 2010

3. Chan TH, Qamra A, Tan KT, Guo J, Yang H, Qi L, Lin JS, $\mathrm{Ng} \mathrm{VH}$, Song Y, Hong $\mathrm{H}$, et al: ADAR-mediated RNA editing predicts progression and prognosis of gastric cancer. Gastroenterology 151: 637-650.e10, 2016.

4. Hironaka S, Sugimoto N, Yamaguchi K, Moriwaki T, Komatsu Y, Nishina T, Tsuji A, Nakajima TE, Gotoh M, Machida N, et al: S-1 plus leucovorin versus S-1 plus leucovorin and oxaliplatin versus $\mathrm{S}-1$ plus cisplatin in patients with advanced gastric cancer: A randomised, multicentre, open-label, phase 2 trial. Lancet Oncol 17: 99-108, 2016.

5. Esteller M: Non-coding RNAs in human disease. Nat Rev Genet 12: 861-874, 2011.

6. Muers M: RNA: Genome-wide views of long non-coding RNAs. Nat Rev Genet 12: 742, 2011.

7. Yan Y, Han J, Li Z, Yang H, Sui Y and Wang M: Elevated RNA expression of long non-coding HOTAIR promotes cell proliferation and predicts a poor prognosis in patients with diffuse large B cell lymphoma. Mol Med Rep 13: 5125-5131, 2016.

8. Chang L, Li C, Lan T, Wu L, Yuan Y, Liu Q and Liu Z: Decreased expression of long non-coding RNA GAS5 indicates a poor prognosis and promotes cell proliferation and invasion in hepatocellular carcinoma by regulating vimentin. Mol Med Rep 13: 1541-1550, 2016.

9. Luo G, Wang M, Wu X, Tao D, Xiao X, Wang L, Min F, Zeng F and Jiang G: Long non-coding RNA MEG3 inhibits cell proliferation and induces apoptosis in prostate cancer. Cell Physiol Biochem 37: 2209-2220, 2015.

10. Gutschner T and Diederichs S: The hallmarks of cancer: A long non-coding RNA point of view. RNA Biol 9: 703-719, 2012.

11. Baldinu P, Cossu A, Manca A, Satta MP, Sini MC, Palomba G, Dessole S, Cherchi P, Mara L, Tanda F and Palmieri G: CASC2a gene is down-regulated in endometrial cancer. Anticancer Res 27: 235-243, 2007.
12. Wang P, Liu YH, Yao YL, Li Z, Li ZQ, Ma J and Xue YX: Long non-coding RNA CASC2 suppresses malignancy in human gliomas by miR-21. Cell Signal 27: 275-282, 2015.

13. Li P, Xue WJ, Feng Y and Mao QS: Long non-coding RNA CASC2 suppresses the proliferation of gastric cancer cells by regulating the MAPK signaling pathway. Am J Transl Res 8: 3522-3529, 2016.

14. Hermanek P, Hutter RV, Sobin LH and Wittekind C: International union against cancer. Classification of isolated tumor cells and micrometastasis. Cancer 86: 2668-2673, 1999.

15. Livak KJ and Schmittgen TD: Analysis of relative gene expression data using real-time quantitative PCR and the 2(-Delta Delta C(T)) method. Methods 25: 402-408, 2001.

16. Lee J, Song J, Kwon ES, Jo S, Kang MK, Kim YJ, Hwang Y, Bae H, Kang TH, Chang S, et al: CTHRC1 promotes angiogenesis by recruiting Tie 2 -expressing monocytes to pancreatic tumors. Exp Mol Med 48: e261, 2016.

17. Huang G, Wu X, Li S, Xu X, Zhu H and Chen X: The long noncoding RNA CASC2 functions as a competing endogenous RNA by sponging miR-18a in colorectal cancer. Sci Rep 6: 26524, 2016.

18. Ponting CP, Oliver PL and Reik W: Evolution and functions of long noncoding RNAs. Cell 136: 629-241, 2009.

19. Lee JT: Epigenetic regulation by long noncoding RNAs. Science 338: 1435-1439, 2012.

20. Batista PJ and Chang HY: Long noncoding RNAs: Cellular address codes in development and disease. Cell 152: 1298-1307, 2013.

21. Zhu X, Tian X, Yu C, Shen C, Yan T, Hong J, Wang Z, Fang JY and Chen $\mathrm{H}$ : A long non-coding RNA signature to improve prognosis prediction of gastric cancer. Mol Cancer 15: 60, 2016.

22. Huang B, Song JH, Cheng Y, Abraham JM, Ibrahim S, Sun Z, Ke X and Meltzer SJ: Long non-coding antisense RNA KRT7-AS is activated in gastric cancers and supports cancer cell progression by increasing KRT7 expression. Oncogene 35: 4927-4936, 2016.

23. Li H, Yu B, Li J, Su L, Yan M, Zhu Z and Liu B: Overexpression of 1ncRNA H19 enhances carcinogenesis and metastasis of gastric cancer. Oncotarget 5: 2318-2329, 2014.

24. Zhao Y, Guo Q, Chen J, Hu J, Wang S and Sun Y: Role of long non-coding RNA HULC in cell proliferation, apoptosis and tumor metastasis of gastric cancer: A clinical and in vitro investigation. Oncol Rep 31: 358-364, 2014.

25. Xie M, Nie FQ, Sun M, Xia R, Liu YW, Zhou P, De W and Liu XH: Decreased long noncoding RNA SPRY4-IT1 contributing to gastric cancer cell metastasis partly via affecting epithelial-mesenchymal transition. J Transl Med 13: 250, 2015. 